\title{
Voltage Regulation in Weak Distribution Grids Using Transformerless Series Compensators
}

\author{
Senthooran Balasubramaniam, Grazia Todeschini, and Petar Igic \\ College of Engineering \\ Swansea University \\ Wales, UK
}

\begin{abstract}
With increasing penetration of distributed generators (DGs), the existing distribution grids are growing weak. Thus, the voltage regulation in distribution grids impose a significant challenge. This can be improved by introducing additional control apparatuses into the existing grid. In this paper, a transformerless static synchronous series compensator (T-SSSC) has been adopted to maximise the power transfer between DGs and a weak grid. A new control strategy has been introduced to reduce the number of switching events during the operation of a $T$ SSSC. Furthermore, a new droop based control scheme has been proposed to minimise the voltage unbalance between phases. A medium voltage distribution grid with embedded T-SSSCs modelled in Simulink has been used to verify the effectiveness of the proposed control schemes. For completeness, the performance a T-SSSC has been investigated following an upheaval in grid short circuit level.
\end{abstract}

Keywords-distributed generation, H-bridge, static synchronous compensator, voltage unbalance, weak grid

\section{INTRODUCTION}

The European Union is planning on reducing the greenhouse gas emissions by $80 \%$ of the 1990 levels by 2050 . However, the generation of greenhouse gases, in particular carbon dioxide, increases in proportion with power demand. Therefore, in order to achieve the low carbon transition, a large amount of renewable energy must be integrated into the existing power grids. Interconnection of renewable energy sources to the power grid is achieved through the use of power electronic converters at transmission and distribution levels.

In recent years, development and deployment of PV systems have been continuously increasing and expected to continue further. At the same time, the increasing number of power electronic converters makes the system more decentralized and vulnerable. The low short circuit capacity could drastically affect the dynamics of the grid-connected inverters. This could trip many DGs from the network at same moment. Thus, the generation seen by the distribution network could significantly reduce. Therefore, PV systems are expected to provide additional ancillary services such as reactive power support, active filtering and load levelling. However, in Europe, inverter design is mainly driven by IEC standards where the vendors are not required to provide any ancillary services. Furthermore, providing additional ancillary services could reduce the lifetime of PV inverters and could be expensive as it curtailed the available PV power. To end this the distributed network operator (DNO)

\footnotetext{
The authors are with the College of Engineering, Swansea University, SA1 $8 \mathrm{EN}$, Wales, UK
}

acts as a governing statute to control system power flow and to improve system stability by placing various control apparatuses in the grid.

The selection of a suitable compensator for improving the quality of energy in modern power system remains a challenge. In recent years, many research activities have focused on the applications of static synchronous compensator (STAT$\mathrm{COMs}$ ) in distribution grids $[\mathrm{x}]$. In contrary, the application of series FACTs controllers in distribution grid has not been extensively studied. Although, STATCOMs are widely used in distribution grids to provide various ancillary services, they may not provide the feasible solution in some cases. The STATCOMs are recommended in a grid with high current harmonic distortion, low SCR, whereas, series controllers are recommended on a system with low power factor, low current harmonic distortion and low SCR. This paper aims to bridge this gap by discussing the application of series controllers for weak distribution grids. In this paper, a transformerless static synchronous series compensator (T-SSSC) topology is adopted increase the hosting capacity of a weak distribution grid. A noval switching methodology is proposed to minimise the losses by reducing the number of switching events. A Simulink based model is used to demonstrate the effectiveness of the proposed control scheme. In addition, a droop control scheme is proposed to limit the unbalance between phases.

\section{H-BRIDGE BASED SERIES COMPENSATOR}

The series flexible FACTs controllers are often used in transmission systems to alter the line power flow. They exhibit lower power losses and cost compared to their shunt counterparts. Among the series FACTs controllers, the gate-controlled series capacitor (GCSC) provide a better solution compared to other more complex and expensive FACTS devices, such as TCSC and SSSC due its simplicity. The operating range of a GCSC is mainly determined by its capacitor size and the phase current magnitude. Therefore, the maximum voltage limited by reactance of the equipped capacitor and line current. To overcome the shortcomings of a GCSC, in [x], transformerless static synchronous series compensator (T-SSSC) is proposed. A T-SSSC could inject the rated voltage even with low phase currents by increasing the dc voltage across its capacitor.

Fig. 1 shows the schematic diagram of a T-SSSC. The device consists of an H-bridge and a dc capacitor. Terminals $T_{1}$ and $T_{2}$ are connected in series with an ac line. A dc voltage $\left(\left(V_{c}\right)\right)$ is established on the capacitor in order to provide a controllable ac voltage source $\left(V_{c o m}\right)$ for the voltage manipulation. 


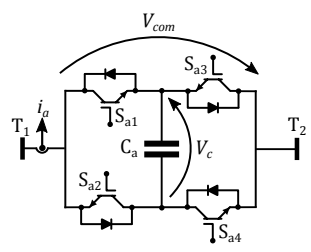

Fig. 1. H-bridge based SSSC.

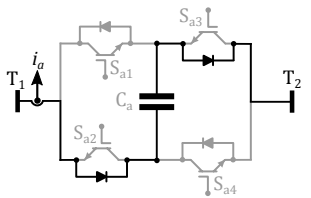

(a)

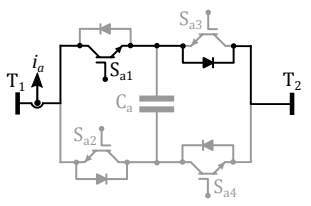

(c)

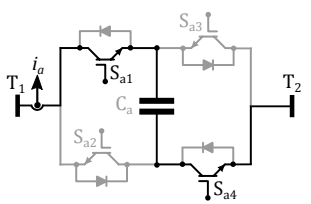

(e)

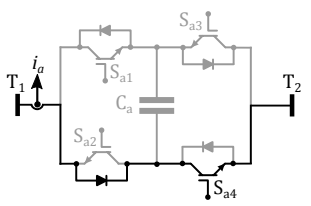

(g)

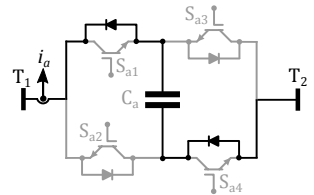

(b)

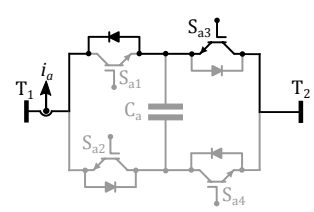

(d)

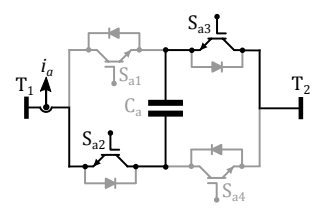

(f)

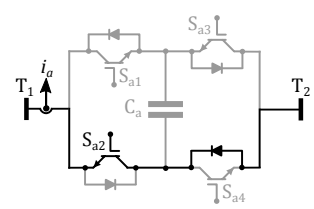

(h)
Fig. 2. T-SSSC operational modes: a) bypass mode 1- $i_{a}>0$; b) bypass mode $1-i_{a}<0$; c) bypass mode $2-i_{a}>0$; d) bypass mode $2-i_{a}<0$; e) charge mode $-i_{a}>0$; f) charge mode $-i_{a}<0$; g) discharge mode $-i_{a}>0$; h) discharge mode $-i_{a}<0$;

\section{A. Operation}

The operational stages of the compensator are shown in Fig. 2. The switching states of the controlled switches and the corresponding changes on the compensator voltages are shown in Fig. 3. When current, $i_{a}$, flows from terminals $T_{1}$ to $T_{2}$ (between $t_{0}-t_{1}$ ), only switch $S_{a 1}$ is modulated, while switches $S_{a 2}, S_{a 3}$ and $S_{a 4}$ are maintained off. When switches $S_{a 1}$ is off (Fig. 2(a)), the line current flows through the naturally commutated diodes and charge the capacitor rapidly. For simplicity, lets assume that the average voltage, $\bar{v}_{c}$, across the capacitor remains a constant. Thus, a positive voltage, $+\bar{v}_{c}$, inserted in series with the ac line. The capacitor is bypassed by turning on switch $S_{a 1}$ (Fig. 2(c)). Between $t_{1}-t_{2}$, the line is reversed and current flows from terminals $T_{2}$ to $T_{1}$. To maintain the power balance, capacitor must be discharged. This is achieved by switching on both $S_{a 2}$ and $S_{a 3}$ (see Fig. 2(f)). During this period, a positive voltage, $+v_{c}$, is inserted in series with the ac line. To minimise the switching losses, between $t_{1}-t_{2}$, switch $S_{a 2}$ maintained on. Therefore when switch $S_{a 3}$ is off, the compensator enters the bypass mode as shown in
Fig. 2(g). The combination of switching states, a, c, f and $\mathrm{g}$ generates the positive half cycle of the inserted ac voltage $v_{\text {com }}$ across terminals $T_{1}$ and $T_{2}$. Similarly, combination of switching states, $\mathrm{b}, \mathrm{d}$, e and $\mathrm{h}$ generates the negative half cycle of the inserted ac voltage $v_{\text {com }}$.

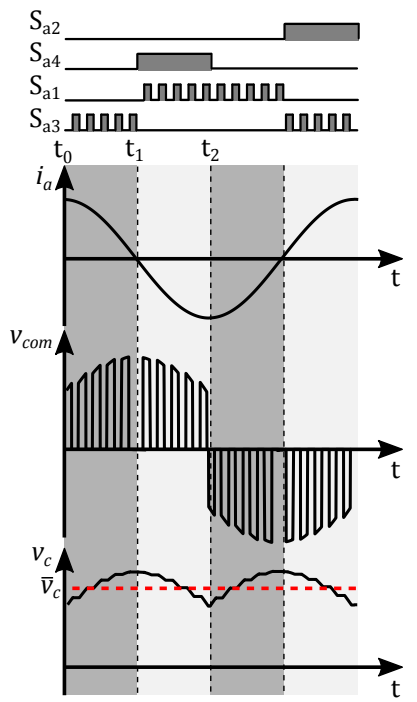

Fig. 3. Compensator switching states.

\section{B. Control System Design}

Fig. 4 depicts the series compensator control scheme. The control system consists of a capacitor voltage controller and a voltage droop controller. A proportional-integral controller is used to realise the capacitor voltage controller. The droop control system uses a proportional gain, $k_{d}$, to calculate the required capacitor voltage, $v_{c, r e f}$, for the series compensation. The droop is enabled when the deviation in PCC voltage exceeds a specific margin $\left|\Delta v_{t h}\right|$. In Fig. 4 , duty cycle $D_{1}$ is used drive switches $S_{a 1}$ and $S_{a 2}$, while $D_{2}$ is used to drive switches $S_{a 2}$ and $S_{a 4}$.

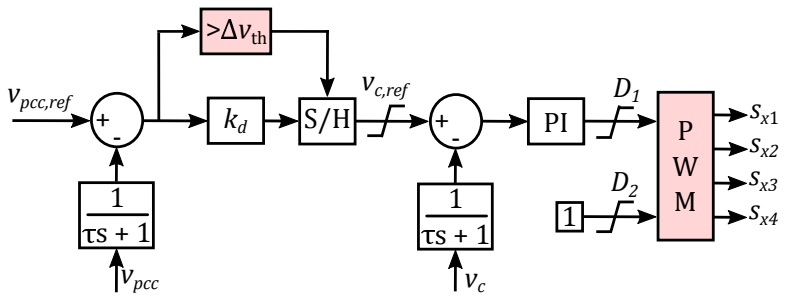

Fig. 4. Compensator controller.

\section{RESULTS}

A. Voltage compensation during weak grid condition

The impact of series voltage compensation in weak grid is studied on a distribution system as shown in Fig. 5. An aggregated pv model is used to represent the local low voltage pv network. A star-star step down transformer is used to connect the DG to the local $11 \mathrm{kV}$ network.

Fig. 6 shows the grid response following a change in power generation without series compensation. A set of vector diagrams is shown in Fig. 7(a) to demonstrate the impact of 


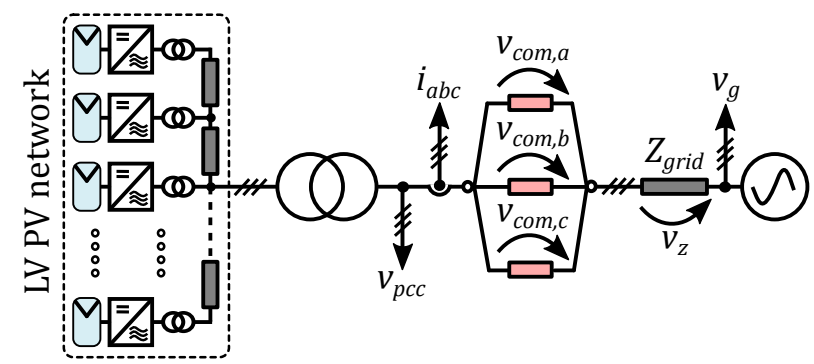

Fig. 5. Distribution grid with aggregated pv network

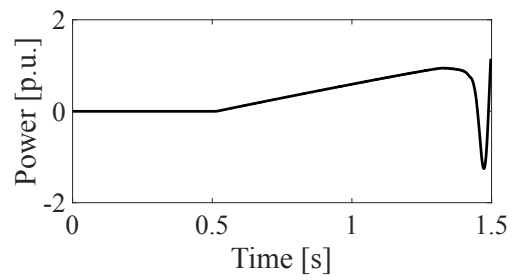

(a)

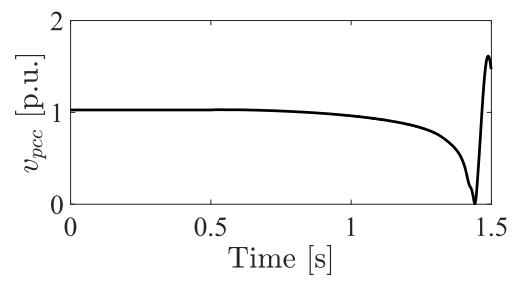

(b)

Fig. 6. System response without series compensation under weak grid condition: (a) power (b) $v_{p c c}$.

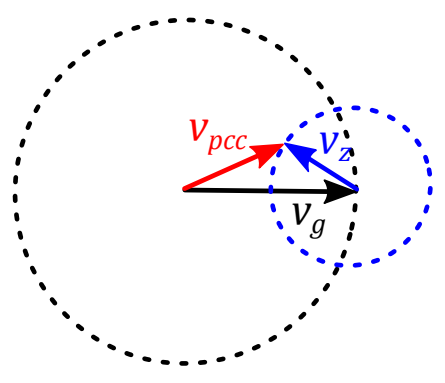

(a)

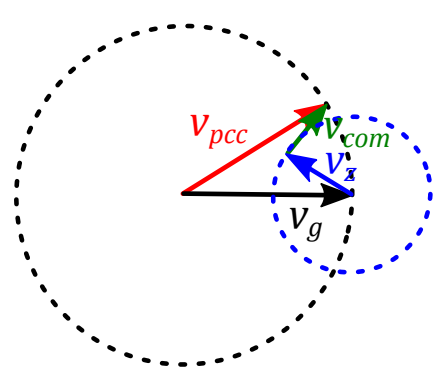

(b)
Fig. 7. PCC voltage vector under weak grid: (a) without compensation (b) with compensation

series voltage compensation on PCC voltage. In Fig. 7(a), grid voltage $v_{g}$ is fixed to 1 p.u. (black circle). At $t=0.5 \mathrm{~s}$, voltage drop $\left(v_{z}\right)$ across the grid impedance starts to rise as more power is injected into the grid. Conversely, the phase angle separation between $v_{g}$ and $v_{z}$ starts to decrease. Thus, the PCC voltage $v_{p c c}$ collapses and trips the converters.

This is resolved by inserting a voltage source in series with each phase. Fig. 8 shows the grid response with series compensation. Following the change in power generation at $t=0.5 \mathrm{~s}$, the PCC voltage started to decrease. At $\mathrm{t}=\mathrm{xs}$, the undervoltage is detected and compensator is requested increase the PCC voltage. Fig. 9 shows the voltage profile of the series compensator. The capacitor voltage is reached a maximum of 0.21 p.u (see Fig. 9(a) ) during the compensation. As demonstrated by the vector illustration in Fig. 7(b), PCC

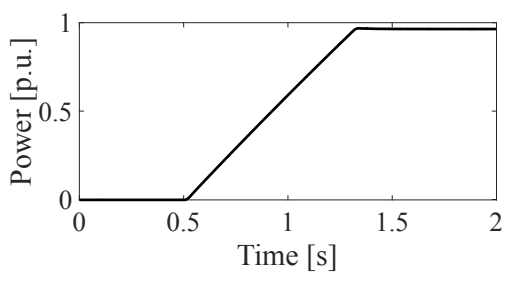

(a)

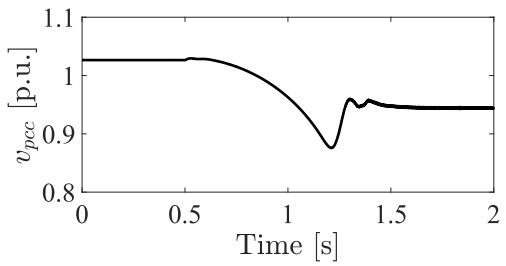

(b)

Fig. 8. System response with series compensation under weak grid condition: (a) power (b) $v_{p c c}$.

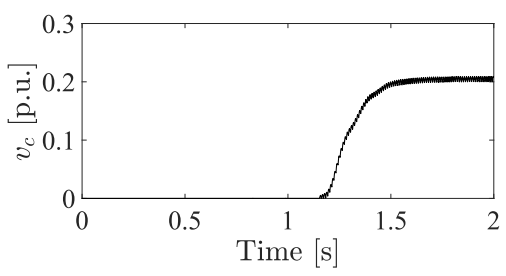

(a)

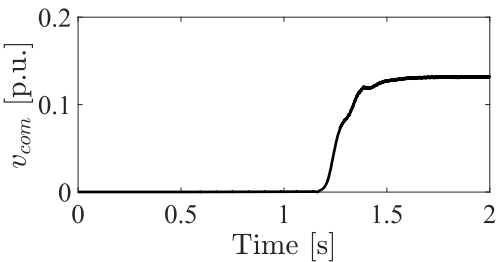

(b)

Fig. 9. Compensation voltage profile: (a) capacitor voltage (b) inserted ac voltage.

voltage is maintained within permissible limit by injecting a voltage $\left(v_{\text {com }}\right)$ (see Fig. 9(b))in series with each phase.

\section{B. Voltage compensation during unbalanced operation}

Unlike transmission systems, single phase DGs are permitted in distribution grids. According to Engineering Recommendation G99 handbook, the maximum aggregate capacity of a single-phase DG module is limited to $17 \mathrm{~kW}$ whereas a three phase DG capacity is limited to $34 \mathrm{~kW}$ [x]. Increasing number of single phase DGs could lead to voltage unbalance between phases. Especially, in presence of a weak grid, voltage unbalance between the phases could significantly increase. The level of voltage unbalance at the PCC should be no greater than $1.3 \%$ for systems with a nominal voltage below $33 \mathrm{kV}$ [x].

In this test case, series compensators are used to limit the unbalance between phases within a permissible margin. Fig. 10 shows the modified compensator control system with an unbalance droop controller. The proposed droop scheme calculates the additional required by comparing the phase voltage against the reference $v_{u b, r e f}$. The droop is activated by signal $S_{e n}$ when the unbalance on phases exceeds the 


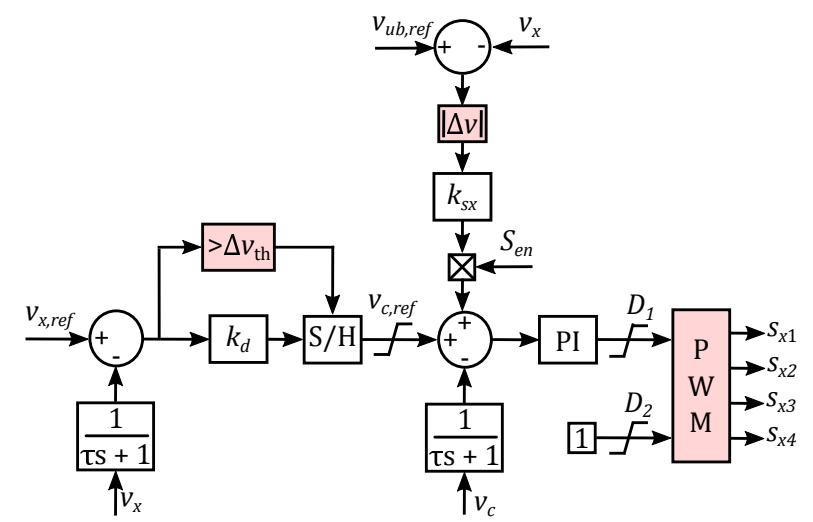

Fig. 10. Compensator controller (refer to Fig. 4) with unbalance droop control.

minimum threshold of $1.3 \%$. The values of $v_{u b, r e f}$ and $S_{e n}$ are determined by a supervisory control system.

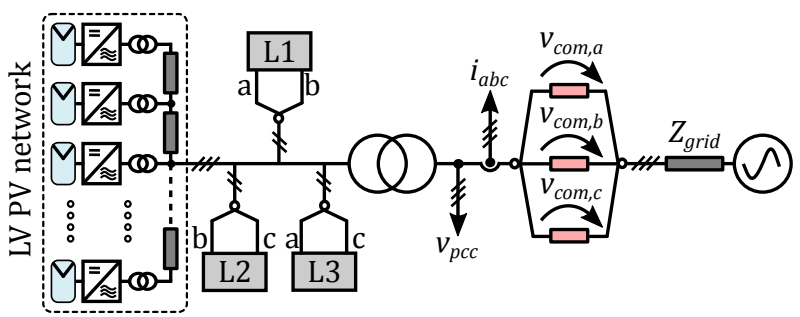

Fig. 11. Distribution grid with unbalanced power generation

Fig. 11 shows test system under study. An unbalance condition is generated by connecting single phase loads L1, L2 and L3 to the secondary side of the star-star transformer. Figs. 12 PCC phase voltages in an unbalance grid. Table I provides the unbalance between phases with and without series compensation. The unbalance in each phase can be calculated as follow:

$$
\begin{aligned}
& \Delta_{a}=\left|u_{m}-u_{a}\right| \\
& \Delta_{b}=\left|u_{m}-u_{b}\right| \\
& \Delta_{c}=\left|u_{m}-u_{c}\right|
\end{aligned}
$$

where $u_{m}=\frac{u_{a}+u_{b}+u_{c}}{3}$ and $u_{a}, u_{b}$ and $u_{c}$ are the magnitudes of phases $a, b$ and $c$, respectively.

Initially, an unbalance of $1.4 \%, 0.6 \%$ and $1.9 \%$ are observed on phases $a, b$ and $c$, respectively. At $t=0.5 \mathrm{~s}$, commentators on phases $b$ and $c$ are trigged to reduce the unbalance. Fig. 11 shows the inserted ac voltage in series with each phase. It has been observed that the unbalance on each phases is maintained within $1.3 \%$ by compensating phases $b$ and $\mathrm{c}$.

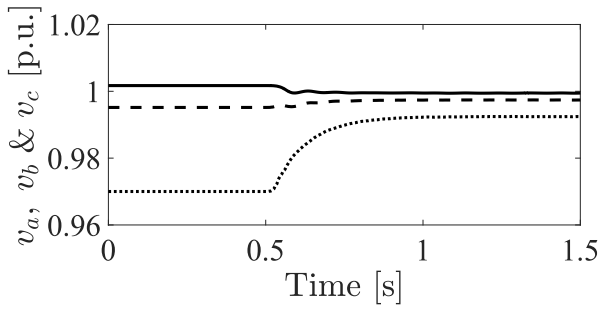

Fig. 12. PCC voltage with unbalance controller

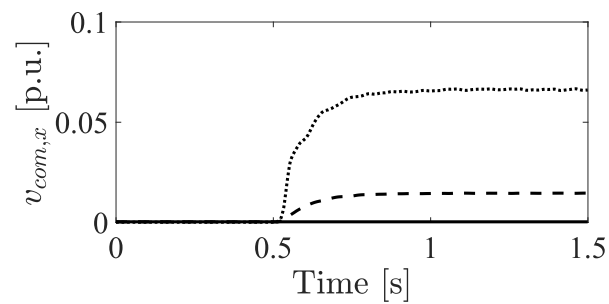

Fig. 13. Compensator voltage profile.

TABLE I. UnBALANCE BETWEEN PHASES [\%]

\begin{tabular}{ccc}
\hline & No comp & with comp \\
\hline$\left|u_{m}-u_{a}\right|$ & 1.4 & 0.4 \\
\hline$\left|u_{m}-u_{b}\right|$ & 0.6 & 0.2 \\
\hline$\left|u_{m}-u_{c}\right|$ & 1.9 & 0.3 \\
\hline
\end{tabular}

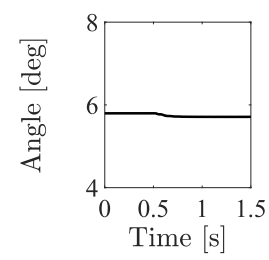

(a)

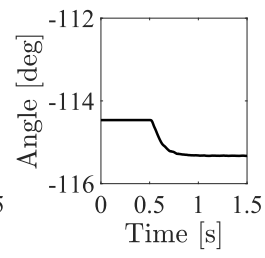

(b)

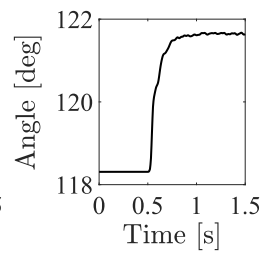

(c)
Fig. 14. Phase angles: (a) Phase $a$; (b) Phase $b$; (c) Phase $c$.

C. Voltage compensation following a sudden change in SCR

In this test case, performance of the proposed control scheme is tested following a transient event. An electrical circuit as shown Fig. 15 is used to emulate a sudden change in grid impedance. Fig. 16 shows the PCC voltage. At $t=0.5 \mathrm{~s}$, the grid impedance is doubled by opening switch $S_{f}$. Following the change in grid impedance, the PCC voltage starts to decrease. Following the undervoltage detection at $t=0.56 \mathrm{~s}$, the compensator is activated and the PCC voltage is increased by in injecting a voltage as shown in Fig. 17 in series with each phase.

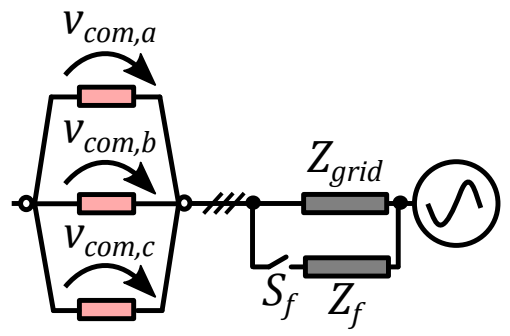

Fig. 15. Distribution grid with variable grid impedance

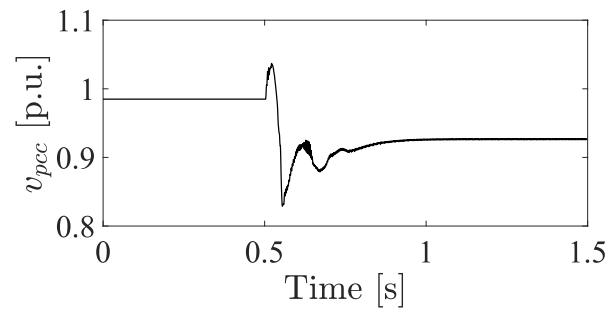

Fig. 16. PCC voltage with compensation. 


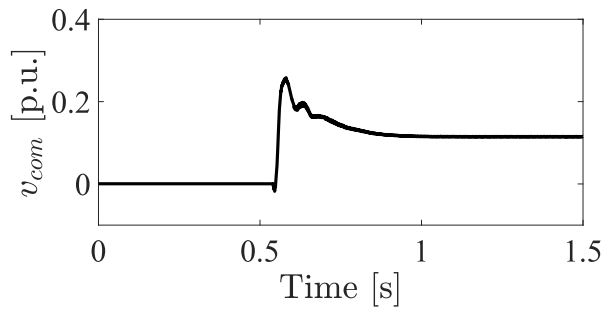

Fig. 17. Inserted series ac voltage.

\section{CONCLUSIONS}

In this paper, the operation and control of a transformerless static synchronous series compensator (T-SSSC) has been discussed for weak grid applications. It has been demonstrated that power exchange between grid tied pv inverters and a weak grid can be a maximised by introducing a T-SSSC into the network. In addition, the proposed droop control scheme is capable of maintaining grid's voltage stability following a sudden disturbance in the grid.

\section{REFERENCES}

[1] 